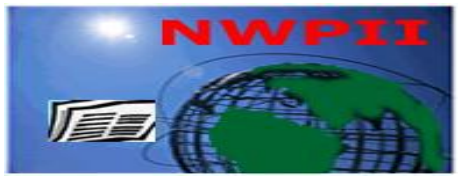

\title{
Effects of Underwater Environment on Different Body Functions
}

\author{
Eman S. Abdul Hamid ${ }^{1}$ and Khaled A. Abdel-Sater ${ }^{2}$ \\ ${ }^{1}$ Student, Qena Faculty of Medicine, South Valley University, Qena, Egypt \\ ${ }^{2}$ Department of Physiology, Faculty of Medicine, Al-Azhar University, Assiut, Egypt \\ "Corresponding Author \\ Khaled A. Abdel-Sater, \\ Department of Physiology, \\ Faculty of Medicine, Al-Azhar University \\ Egypt \\ Tel:00201067970804 \\ E-mail:Khaled 71111@yahoo.com
}

Received:08 September 2020; | Revised:10 April 2021; | Accepted: 19 April 2021

\begin{abstract}
Physiology is the study of life processes within the body. This review provides an overview of how the human body normally responds to the varied conditions underwater. This is a brief overview of respiratory, cardiovascular and sensations physiological reactions associated with diving. Breathing rate, gas exchange, lung volumes, heart rate, arterial blood pressure, cardiac output, renal functions, gut effects, regulation of temperature, vision, hearing, equilibrium, smell and taste, and touch were mentioned.
\end{abstract}

Keywords: Underwater, Diving, Diving reflex, Dive response, Dive physiology

\section{Introduction}

Underwater or diving at various depths in water elicits profound physiological responses. The purpose of this review is to outline the physiological responses associated with diving. An understanding of these physiological contributes to diving safety and enables a diver to describe diving-related medical symptoms when problems occur. A knowledge of diving physiology enhances the safety and enjoyment of sports diving.

\section{Respiratory Functions during Diving}

Adequate exchange of oxygen and carbon dioxide while diving requires the ability to maintain ventilation in the face of significantly increased resistive and elastic loads. An increase in resistive, elastic, and inertial components is induced by diving. The primary effect of diving on resistance is mediated by the proportional increase in breathing gas density with the depth of immersion. This occurs because breathing underwater can only occur if breathing gas is delivered to the diver at a pressure within a few $\mathrm{cmH} 2 \mathrm{O}$ of the ambient pressure at the diver's depth. For turbulent gas flow, which is present throughout most of the conducting airways, flow resistance is proportional to density. During diving, the external breathing apparatus adds an additional resistive load. Elastic load is enhanced primarily by changes in trans-respiratory pressure. Inertial mechanical load increase has a 
minimal effect on the diver. Added challenges include blunted respiratory drive due to elevated partial pressures of inert gas and oxygen, and possibly impaired diffusion within the alveolus. Lung compliance decreases due to the increased central blood volume during immersion as it engorges and stiffens lung tissues. Also, it compresses small airways, elevating airway resistance; and increases air trapping and closing volume. These alternations result in an increase in the load on respiratory muscles ${ }^{[1]}$. Adequate ventilation and gas exchange have been achieved in spite of the above opposing forces because of the increase in central blood volume and elimination of blood flow dependence on gravity, thus promoting homogeneous perfusion of the lungs ${ }^{[2]}$.

As depth increases, ambient pressure at specific depth increases by 1 bar for each 10 meters underwater. Pressure doesn't affect fluids or solid parts of the body as they are not compressed but affects cavities filled with gases as they are compressible ${ }^{[3]}$. Water immersion does not affect the tidal volume or residual volume $(\mathrm{RV})$. It decreases vital capacity by about 5 to $10 \%$ mainly because of a decrease in expiratory reserve volume. Several factors contribute to decreased lung volumes during immersion; among these; the chest ward pooling of blood (a redistribution of 500-800 $\mathrm{ml}$ of blood from the legs into the large veins and pulmonary vessels) and chest ward displacement of the diaphragm appears most important ${ }^{[4]}$. If the diver descends far enough for lung volume to shrink to $\mathrm{RV}$, the alveolar pressure will be some $40 \mathrm{cmH} 2 \mathrm{o}$ less than body surface pressure and the chest wall will be very stiff. It should be noted that at a depth of $99 \mathrm{ft}$ the total lung capacity has been reduced to the RV. Thus a diver entering the water in full inspiration will be in a state equivalent to full expiration without having lost any air when he has dived to $99 \mathrm{ft}$. If the diver continued to go deeper, (1) the air in the lungs would remain at constant volume; (2) pressure and the pressure differential in the alveoli would increase, causing a transfer of fluid from the alveolar capillaries into the lungs (pulmonary edema); (3) as the descent was continued, vessels would burst, resulting in pulmonary hemorrhage; and finally, (4) ribs would crack and the chest wall would cave in. Therefore, human diving to depths greater than $99 \mathrm{ft}$ must be protected by a positive-pressure breathing apparatus of some kind ${ }^{[1]}$.

The work of breathing also increases underwater ${ }^{[5]}$. A change in breathing pattern in endurance underwater swimming is recorded. Fifteen minutes after the start of constant exercise an abrupt $20-25 \%$ increase in ventilation has been observed ${ }^{[6]}$. This is due to respiratory compensation for metabolic acidosis, and possibly respiratory muscle fatigue ${ }^{[7]}$.

\section{Hyperbaric effects on Cardiovascular System}

An increase of oxygen partial pressure of breathing gas in proportion to depth causes cardiovascular changes ${ }^{[8]}$. Relative bradycardias have been reported to occur in humans underwater [9]. The heart rate can drop to $20-30 \mathrm{~b} / \mathrm{min}$ while diving. Bradycardia is often complicated by cardiac arrhythmia ${ }^{[8]}$. During diving, not only is the pacemaker activity of the SA node altered, but sometimes the cardiac contraction also seems to be initiated by the ventricle ${ }^{[10]}$. The incidence of cardiac arrhythmias during diving increases significantly when the water temperature is lower ${ }^{[8]}$.

Bradycardia is partially caused by the increased $\mathrm{PO}_{2}$ (oxygen-dependent) but to a greater degree is caused by other non-oxygen-dependent factors related to the increased inert gas pressure ${ }^{[10]}$. Oxygen-dependent bradycardia is caused by different mechanisms. It is mainly due to the direct effect of high oxygen pressure on the myocardium, combined to a lesser extent to the parasympathetic effect. The parasympathetic effect occurs as hyperoxia induces vasoconstriction causing arterial hypertension, which in turn stimulates baroreceptors ${ }^{[11]}$. The non-oxygen-dependent bradycardia was caused primarily by a reduced $\beta$-adrenergic stimulation of the heart. Non-oxygen-dependent hyperbaric bradycardia occurs due to both density increase of the inspired gas and ambient pressure increase ${ }^{[10]}$. Other mechanisms include the altered respiratory pattern which may modify the heart rate secondarily. The other is circulatory deconditioning under hyperbaric environments, which masks the hyperbaric bradycardia ${ }^{[9]}$. Slowing the heart rate reduces cardiac oxygen consumption, and compensates for hypertension due to vasoconstriction ${ }^{[8]}$. 
The effects of hyperbaric oxygen on arterial blood pressure have been controversial. Many authors have reported that an increase in systolic and diastolic blood pressure occurs, other authors found no alteration or non-significant changes. The increase arterial blood pressure was reported to values as high as $280 / 200 \mathrm{mmHg}$. This an increase may be due to an increase in peripheral vascular resistance caused by hyperoxic vasoconstriction. The effect on arterial pressure can be of variable intensity because of the localization of the hyperoxic vasoconstriction (arteries of a smaller diameter than the medium- and small-size arteries responsible for the major part of the arterial resistance), of the anatomical and physiological differences related to regional circulation in the organs, of whether the perfused areas are hyperoxic or not, the overall consequences of the vasoconstriction may be very variable. Also, variation in cardiac output has an influence on arterial blood pressure ${ }^{[11]}$.

Bradycardia, apnea, and increased peripheral vascular resistance in response to immersion are collectively referred to as the diving reflex ${ }^{[12]}$. The intensity of this reflex is more pronounced in colder water. The splenic contraction has been shown to occur in humans and likely plays a role in supplying additional $\mathrm{O}_{2}$-rich blood to the vital organs of the body ${ }^{[13]}$.

A decrease in cardiac output was observed on exposure to hyperbaric oxygen, but the amplitude was small (10-15\%) and the physiological significance uncertain ${ }^{[11]}$. Cardiac output decrease is caused by hyperoxic-induced bradycardia. However, a slightly diminished cardiac contractility may also be a contributing factor ${ }^{[14]}$. A combined decrease in systolic ejection volume has been reported. An increase of $11 \%$ of myocardial contractility when $\mathrm{PO}_{2}$ was increased to $460 \mathrm{mmHg}$ was reported due to a direct beneficial effect of oxygen on the myocardial fibre. Preload increases due to the increase in ventricular filling time caused by the bradycardia, and a decrease in ventricular compliance which is made evident by the pressure / volume curves (i.e., Starling curve). The increasing effect on preload was mainly related to the hyperbaric pressure rather than to the hyperoxia. Ventricular afterload increases due to the hyperoxic arterial vasoconstriction. Increase of both preload and afterload results in a balance between them ${ }^{[11]}$.

Regional blood flow is regulated by the metabolic requirements of the respective organ system. Within $5 \mathrm{~min}$ of exposure to 3.5 atmospheres absolute (ATA) oxygen, a 25\% decrease in the cerebral blood flow was reported. This is accompanied by an increase in cerebral vascular resistance by $55 \%{ }^{[8]}$. Short-term hyperoxia causes increased cerebral vasoconstriction and further the reduction of blood flow. However, even with the reduction of cerebral blood flow, the cerebrum receives more oxygen than it would otherwise ${ }^{[14]}$. An increase in cerebral blood flow was suggested to be dependent on an increase in cerebral $\mathrm{PCO}_{2}$ induced by hyperoxia. As it caused (1) alveolar hypoventilation (due to a decrease in the respiratory drive) (2) decrease in carbaminohaemoglobin (Haldane effect) (3) decrease in cerebral blood flow due to hyperoxic vasoconstriction, so that vasodilation appeared. This was countered by the vasoconstricting effect of the hyperoxia ${ }^{[11]}$.

Hyperbaric oxygenation decreases total coronary blood flow. This was combined with a decrease in myocardial $\mathrm{VO}_{2}$ of around $20 \%$ and a decrease in lactate extraction ${ }^{[11]}$. This diminished do not seem to be associated with a primary alteration in myocardial contractility, but rather may result from physiologic autoregulation of the myocardium to increasing levels of $\mathrm{PaO}_{2}$ as, with increasing levels of oxygenation, coronary blood flow and myocardial oxygen consumption were all significantly reduced, while intrinsic myocardial function, as measured by the stroke-work/enddiastolic volume relationship was unchanged ${ }^{[23]}$. The coronary blood flow is closely adjusted to the myocardial $\mathrm{VO}_{2}$, as the myocardium cannot increase its capacity for oxygen extraction which is already maximal ${ }^{[11]}$.

Hepatic blood flow has been shown to be maintained even for 16-hour exposures at 1.5 ATA as the greater part of the hepatic blood flow is of portal venous origin and that the increase in PO2 in the portal venous blood is much smaller than in the arterial blood. Thus hyperoxia causes vasoconstriction of the hepatic artery resulting in an increase in portal blood flow as a compensatory mechanism for maintaining hepatic blood flow ${ }^{[11]}$. 
Muscular blood flow depends on the level of muscular activity. Blood flow to abdomen and thorax muscle increases in hyperbaric hyperoxia, and in hyperbaric normoxia. This is probably due to the increase in $\mathrm{VO}_{2}$ induced by the increase in muscular work. An increase in muscular work is due to an increase in work of breathing due to gas density-related ventilation resistance under hyperbaric condition ${ }^{[11]}$. The reduction in limb blood flow is due to vasoconstriction resulting from increased activity of the sympathetic nerves supplying arteries in the arms and legs. Blood is shifted to the body' $s$ centre to maintain normal body core temperature and to ensure adequate perfusion of organs (centralization) ${ }^{[13]}$.

\section{Effects of Immersion on Renal Functions}

Immersion causes both a water diuresis and a natriuresis, which are apparently controlled by different mechanisms. Although the increase in diuresis is usually manifest by the first or second hour, the natriuresis usually peaks by the fourth or fifth hour ${ }^{[10]}$.

Immersion diuresis occurs due to an increase in central blood volume which results in distention of left atrial or pulmonary vein mechanoreceptors. This results in suppression in anti-diuretic hormone release as a reflex response ${ }^{[10]}$. The renal sympathetic nervous activity was suppressed and it has long been considered a mediator of the renal responses to water immersion ${ }^{[16]}$. There is the possibility that this reduction in renal sympathetic activity is due to mechanoreceptor stimulation, which increases diuresis by altering intrarenal perfusion, and by direct tubular effects [10]. Immersion also produces an augmentation of prostaglandin E (PGE) excretion, which reflects increased renal PGE synthesis ${ }^{[17]}$.

Immersion causes increased natriuresis, but this increase is strongly correlated to salt intake and also varies among individuals. The lack of dependence of this phenomenon on diuresis is evident from the fact that even if the anti-diuretic hormone is administered to prevent diuresis, natriuresis may still an increase in response to immersion ${ }^{[10]}$. Immersion natriuresis occurs due to increase in sodium excretion which results in suppression of renin- angiotensin-aldosterone system ${ }^{[16]}$.

Bicarbonate excretion is increased during immersion and is associated with an increase in urinary $\mathrm{PCO}_{2}$ and a marked increase in urinary $\mathrm{pH}$. The titratable acid, urinary ammonium, and net acid excretion are suppressed in immersion, effects that are not related to any systematic acid-base balance changes ${ }^{[10]}$.

\section{Diving effects on Gastrointestinal System}

During head-out immersion in the upright position the abdomen, being at greater depth is subjected to a proportionately higher water pressure than the thorax. This pressure is transmitted to the abdominal content. The pressures on each side of the diaphragm changes to be positive on the abdominal side instead of being equal and negative on both sides in the non-immersed state. Thus the abdominal pressure becomes considerably higher than on the thoracic side (by about $15 \mathrm{cmH} 20$ ). This results in displacing of the diaphragm in the cranial direction and the compression of the lungs generating an increase in pleural pressure and therefore in esophageal pressure have shown that although the pressure increased by about $5 \mathrm{mmHg}$, the esophageal pressure went up by about $5 \mathrm{mmHg}$. This creates a marked pressure difference that would tend to push the gastric contents into the esophagus, was it not for the distal esophageal sphincter, whose pressure during immersion also increases and remains up to $15 \mathrm{mmHg}$ higher than gastric pressure ${ }^{[10]}$.

When a diver swims head-down toward the bottom, the vertical pressure differences on his body are reversed in comparison to the head-up posture. Thus, the diver's mouth and breathing regulator are at a greater depth than the lungs, and especially the stomach. The high air pressure in the oral cavity relative to the esophagus may lead to considerable air ingestion. Change in body buoyancy, determined by underwater weighing, was taken as a measure of aerophagia during diving ${ }^{[10]}$.

\section{Regulation of Temperature Underwater}

Due to cardiac changes induced by immersion in water; divers must tolerate and compensate for 
changes temperature ${ }^{[18]}$. Because water has a specific heat approximately 1000 times greater than that of air and a thermal conductivity 25 times greater than that of air, the body loses heat much faster in water than in air of the same temperature [19].

With cold skin and with core temperatures below $36^{\circ} \mathrm{C}$, the defense mechanisms of the body are activated. The hypothalamus regulates temperature in two ways. Most importantly, it reduces heat loss by behavioral changes (clothing, position, room thermostat setting) and generalized vasoconstriction (that decreases the heat conduction from internal organ to skin and also decreases heat loss to surrounding). Second, it causes heat gain by shivering and non-shivering mechanisms. Shivering will occur in most normal persons when the skin is cooled to an average value of $30^{\circ} \mathrm{C}$. It consists of rhythmic, oscillating skeletal muscle contractions that occur at a rapid rate of 10 to 20 per second. All energy liberated during these muscle tremors is converted to heat because no external work is accomplished. Shivering can increase basal body heat production by up to five times. The nonshivering form of thermogenesis is a complex metabolic response to cold, thought to be mediated by the catecholamines and glucocorticoids secretions ${ }^{[20]}$.

Unfortunately, these mechanisms rarely achieve heat balance, so that the diver continues to lose heat ${ }^{[21]}$. Heat loss normally occurs by radiation, conduction, convection, and evaporation. Cutaneous evaporation and radiation play no role. The respiratory heat loss comes from breathing dry, cold air from a tank or surface supply, which the diver breathes, heats, moistens and expires. Thus, evaporation occurs when the air comes in contact with moist lung tissue. This loss is further exacerbated when the diver breathes a mixture of helium and oxygen under pressure. Helium breathed by divers causes heat loss at a rate seven times with a resulting $28 \%$ metabolic heat loss through respiration. Helium provides another difficulty: a layer of heliox next to the skin provides far less insulation than does a layer of air. In addition, body movement in water reduces the thermal insulation of the body shell, thus facilitating heat loss from the core to water during diving ${ }^{[22]}$.
Because of large individual differences in cold tolerance, every diver must determine the most suitable protection on an individual basis. In commercial and military salvage diving, suits are heated with hot water. Wet suits, the most frequently used form of protection, offer limited protection against cold-limited by pressure (below shallow depths their effectiveness is markedly reduced) and also limited by time (the longer the exposure the greater the heat loss that will occur) ${ }^{[18]}$.

Heat imbalance causes complications. These complications are only just being recognized as important, and divers should be aware that the faster the rate of heat loss, the smaller the drop in core temperature for a given quantity of heat loss. Complications of a cold include; hypothermia which in turn may lead to death besides cardiovascular reflex. Cardiac irregularities, impairment of rational thinking, sensory and motor degradation, loss of consciousness, and drowning all occur when body temperature falls below $35^{\circ} \mathrm{C}$ [21].

\section{Underwater Visibility}

As the water is dense than air, it causes poor visibility and sound ${ }^{[23]}$. Underwater, different factors influence what a diver sees. These factors are diffusion, which scatters the light; turbidity, which blocks the light; absorption, which alters the colour and intensity of the light; and refraction, which "bends" the light. Light insufficiency represents the greatest problem underwater ${ }^{[10]}$.

In water, the amount of illumination commonly falls below the levels required for photopic vision, due to extensive absorption by the water. Turbid water would cause even more extreme reduction, of course. Thus divers in clear, deep water or shallow, turbid water frequently do not have enough light for colour vision, unless they carry their light sources. In addition to the overall loss of light, the transmission of light energy by the water changes underwater colour vision. Colours are different wavelengths reflected by an object. Water absorbs colours wavelengths to differing degrees. The longest wavelengths, with the lowest energy, are absorbed first. In clean oceanic water, red is absorbed in the first metre, orange in the first five metres, yellow in the first ten, and green and 
blue at greater depths. This explains why most things, regardless of their colour on the surface, appear to be coloured shades of blue or green at depths beyond about ten metres. Thus the only colour remaining in deep, clear ocean water is bluegreen $^{[24]}$.

The cornea, humours and crystalline lens of the eye together form a lens that focuses images on the retina. The refractive index between the cornea and water is similar (both about 1.33). When one is underwater, the surrounding water on the outside and the aqueous humour inside the eye combine to neutralize the cornea's focusing power (from +43 dioptres to almost +4.3 dioptres). So, instead of focusing images on the retina, they are focused behind the retina, resulting in an extremely blurred image from hypermetropia ${ }^{[10]}$. To avoid this, mask or special glasses are required for providing air interface resulting in two interfaces with different refractive power ${ }^{[18]}$.

Since the refractive index of the water is fourthirds that of air, the optical (apparent) distance of a target underwater is three-fourths of that in air. Immersed objects appear about 30\% larger and closer, due to the distorted perception. (i.e. magnification $=1.33 \times$ ). This distortion derives from the passage of the rays of light from the water into the gas environment within the mask, due to the refraction at the interface where the light speed increases ${ }^{[18]}$. Light speed is $186,000 \mathrm{miles} / \mathrm{sec}$ in air but only $135,000 \mathrm{miles} / \mathrm{sec}$ in water ${ }^{[5]}$. Distortion affects divers perception. Effects include underestimations of distances underwater, overestimations of size, distortions of shape and position, and interference with hand-eye coordination. Interference with hand-eye coordination occurs as objects are not located where they appear to be. Optical distortion affects distance perception but water turbidity has the opposite effect. Studies show that perception disorders improve with experience except for size perception which shows no improvement ${ }^{[10]}$.

Underwater vision is greatly affected by the scattering and absorption of light photons on transmission through water. Scattering occurs as a result of the direction change of photons on hitting particles suspended in the water. Scattering causes loss of contrast between objects and their backgrounds causing contrast to fall below $2 \%$ in water ${ }^{[10]}$.

\section{Auditory Function Underwater}

Hearing is also altered in the water where sound conduction is faster about 25 times than on the air which results in impairment of sound acuity [23].

As the middle ear is a closed air space, an increase of pressure during diving causes the volume to decrease. This may result in pressure problems so pressure equalization must be achieved. This is done through the Eustachian tube which opens as middle ear volume changes. The opening of the Eustachian tube allows air to enter the middle ear for pressure equalization as it connects the middle ear to the throat ${ }^{[8]}$. The impedance mismatch of sound transmission between air and water is overcome by external and middle ear (aircontaining structures) ${ }^{[10]}$.

Air conduction (AC) thresholds were elevated of 30 to $40 \mathrm{~dB}$ in the middle frequencies. This elevation was not related to depth, but no alterations in cochlear function were observed. Bone conduction (BC) thresholds remained unchanged. These effects were believed to be caused by the increased pressure and density of compressed air and the resulting increased impedance of the middle ear transformer and decreased sound conduction to the inner ear ${ }^{[10]}$.

The mechanism of underwater hearing is still not clear. Different theories suggest the mechanism of underwater hearing. One of them is a tympanic theory suggesting a similar mechanism to $\mathrm{AC}$ as sound waves are conducted to the inner ear via the middle ear. Other studies provided evidence supporting an osseous $\mathrm{BC}$ mechanism in which the sound field in the water surrounding the head induces skull bone vibrations and the endocranium contents to the cochlear capsule ${ }^{[8]}$. The underwater hearing at low sound intensities may be attributed to soft tissue conduction or non-osseous $\mathrm{BC}$ in which the sound was transmitted by soft tissue sites on the head, neck, and thorax. At higher intensities, the sound was transmitted by osseous BC mechanisms which involve actual skull bone vibrations. Some theories suggest that both $\mathrm{AC}$ and $\mathrm{BC}$ are involved in underwater hearing ${ }^{[25]}$. 
$\mathrm{BC}$ hearing is, strongly, suggested as $\mathrm{AC}$ hearing exceeds underwater hearing by $30-60 \mathrm{~dB}$ and this indicates that underwater hearing is less efficient as the threshold sound pressure level in the air is much less than in water, the thing that does not occur. The BC threshold is $45-68 \mathrm{~dB}$ below the $\mathrm{AC}$ threshold and this difference is similar to the difference in the AC and underwater hearing [8]. Shupak et al. ${ }^{[26]}$ studied the presence of air around the pinna and inside the external ear canal and found that no improvement of underwater hearing sensitivity or sound localization. These results support the argument that $\mathrm{BC}$ plays the main role in underwater hearing.

\section{Proprioceptive and Equilibrium during Diving}

Underwater, at neutral buoyancy, cues of position received from these senses are markedly reduced or absent. This effect may be exaggerated by the diver's suit and other equipment. Organic cues are still effective since gravity continues to exert its attraction on internal organs, such as the stomach and intestines, but these cues may be minimized by the wearing of a snugly fitting wet suit, scuba tanks, and weight belt. When some of the equilibrium cues are absent or diminished as in many underwater activities, the undisturbed cues become very important. To maintain equilibrium and balance on a fine level, particularly during rapid and intricate body movements, the vestibular sense is thought to be necessary regardless of the number of other cues available ${ }^{[8]}$.

\section{Effects of Diving on Smell and Taste}

Both taste and smell have no significant importance for the diver. A minimal decrease in the threshold for both of them is reported in case of existence for long periods under pressure but, these changes go unnoticed. The human sense of smell depends on esters released by things in our environment. These are airborne molecules that have a certain shape that fits specific olfactory receptors in our nose. While esters are watersoluble to some degree, they do this by bonding with a hydrogen molecule; which changes their shape. This results in the ester not fitting the receptors in our nose, rendering the smell undetectable by humans ${ }^{[8]}$. On exposure to heliox at 18.6 ATA, significant changes in taste sensation have been shown to occur. These changes include: increased sensitivity to sweetness, increased sensitivity to bitter taste, a decline of sour sensitivity, and an decrease in salt sensitivity ${ }^{[27]}$.

\section{Tactile Function Underwater}

Divers may experience two kinds of impairment to their tactile function, one due to being in the water and in a very thick environmental protection suit, the other due to the cold. The water effect seems to be a hindrance, mainly, to motor activity due to some combination of instability, incumbrance of equipment, neutral (approximately) buoyancy, viscous resistance, resistance to limb flexion, and reduced sensory input and, thus, impaired sensory functions. Cold causes losses in sensory and motor function and disrupts cognitive activity, especially when extended in time so that sustained attention and memory are involved ${ }^{[8]}$.

\section{References}

1 Moon RE, Cherry AD, Stolp BW, Camporesi EM. Pulmonary Gas Exchange in Diving. $\boldsymbol{J}$ Appl Physiol. 1985 ; 106(2) : 668-77. DOI:10.1152/japplphysiol.91104.2008

2 Lafay V, Barthelemy P, Comet B, Frances Y, Jammes Y. ECG Changes During the Experimental Human Dive HYDRA 10 (71 atm/7, $200 \mathrm{kPa})$. Undersea Hyperb Med. 1995; 22(1) : 51-60. [PMID: 7742710]

3 Rusoke-Dierich O.Diving Medicine.1st edition. Springer International Publishing. Cham 2018. DOI :10.1007/978-3-319-73836-9

4 Warkander DE, Nagasawa GK, Lundgren CE. Effects of Inspiratory and Expiratory Resistance in Divers' Breathing Apparatus. Undersea Hyperb Med. 2001; 28(2) : 63-73. [PMID: 11908697]

5 Spira A. Diving and Marine Medicine Review Part 1: Diving Physics and Physiology. Journal of Travel Medicine 1999; 6(1), 32. [PMID: 10071371. DOI: 10.1111/j.1708-8305.1999.tb00865.x.]

6 Wylegala JA, Pendergast DR, Gosselin LE, Warkander DE, Lundgren CE. Respiratory 
Muscle Training Improves Swimming Endurance in Divers. Eur J Appl Physiol 2007; 99(4): 393-404. DOI:10.1007/s00421-0060359-6

7 Ray AD, Pendergast DR, Lundgren CE. Respiratory Muscle Training Improves Swimming Endurance at Depth. Undersea Hyperb Med 2008; 35(3): 185-196. [PMID: 18619114]

8 Shilling C.(Ed.). The Underwater Handbook: A Guide to Physiology and Performance for the Engineer. 1st edition, Springer Science \& Business Media. New York and London 2013. [DOI: 10.1007/978-1-4684-2154-5].

9 Lin YC, Shida KK. Mechanisms of Hyperbaric Bradycardia. Chin J Physiol. 1988; 31(1):1-22. [PMID: 3067986]

10 Carlston CB,Mathias, RA, Shilling, CW. (Eds.). The Physician's Guide to Diving Medicine. 1st ed. Springer Science \& Business Media. New York and London 2012. DOI: 10.1007/978-14613-2671-7

11 Mathieu D. (Ed.). Handbook on Hyperbaric Medicine (Vol. 27). 1st edition, Springer. New York: 2006. [DOI:10.1007/1-4020-4448-8]

12 Godek D, Freeman AM. Physiology, Diving Reflex. In: StatPearls. Treasure Island (FL): Stat Pearls Publishing 2019. [PMID: 30855833]

13 Marabotti C,Piaggi P, Menicucci D, Passera M, Benassi A, Bedini R, L'Abbate A. Cardiac Function and Oxygen Saturation During Maximal Breath-Holding in Air and During Whole-Body Surface Immersion. Diving and Hyperbaric medicine 2013; 43(3), 131-137. [PMID: 24122187]

14 Goyal A,Chonis T,Cooper JS. Hyperbaric Cardiovascular Effects. In StatPearls. Treasure Island (FL): StatPearls Publishing 2020. [PMID: 29489293.]

15 Savitt MA, Rankin JS, Elberry JR, Owen CH, Camporesi, EM. Influence of Hyperbaric Oxygen on Left Ventricular Contractility, Total Coronary Blood Flow, and Myocardial Oxygen Consumption in the Conscious Dog. Undersea \& Hyperbaric med. 1994; 21(2): 169-183. [PMID: 8061558]

16 Hammerum MS, Bie P, Pump B, Johansen LB, Christensen NJ, Norsk, P. Vasopressin,
Angiotensin II and Renal Responses during Water Immersion in Hydrated Humans. The Journal of Physiology 1998; 511(1): 323-330. DOI:10.1111/j.1469-7793.1998.323bi.x]

17 Epstein M. Water Immersion and the Kidney: Implications for Volume Regulation. Undersea Biomed Res. 1984;11(2):113-121. [PMID: 6567431]

18 BoscoG, Rizzato A, Moon R E, Camporesi EM. Environmental Physiology and Diving Medicine. Frontiers in psychology 2018; 9: 72. DOI:10.3389/fpsyg.2018.00072]

19 Tipton MJ, Collier N, Massey H, Corbett J, Harper M. Cold Water Immersion: kill or Cure?. Experimental Physiology 2017; 102(11): 1335-1355. [PMID: 28833689. DOI: 10.1113/EP086283]

20 Hoffmann ME,Rodriguez SM, Zeiss DM, Wachsberg KN, Kushner RF, Landsberg L, Linsenmeier RA. 24-h Core Temperature in Obese and Lean Men and Women. Obesity (Silver Spring) 2012; 20(8):1585-90. [PMID: 22240727. DOI:10.1038/oby.2011.380]

21 Foster GE,Sheel AW. The Human Diving Response, its Function, and its Control. Scand J Med Sci Sports. 2005; 15(1): 312.DOI: 10.1111/j.1600-0838.2005.00440.x]

22 Noonan B, Bancroft RW, Dines JS, Bedi A. Heat- and Cold-Induced Injuries in Athletes: Evaluation and Management. $\boldsymbol{J}$ Am Acad Orthop Surg. 2012; 20 (12):744-54. [PMID: 23203934. DOI:10.5435/JAAOS-20-12-744]

23 Luthfi OM.Review of Human Physiology in the Underwater Environment. Health Sciences 2019; 8(8): 117-121. [ISSN No: 2319-5886]

24 Marshall J. Vision and Lack of Vision in the Ocean. Current Biology : CB, 2017; 27(11): R494-R502. DOI:10.1016/j.cub.2017.03.012

25 Chordekar S, Kishon-Rabin L, Kriksunov L, Adelman C, Sohmer H. Experimental Analysis of the Mechanism of Hearing under Water. BioMed Research International 2015; 526708. DOI: $10.1155 / 2015 / 526708$

26 Shupak A,Sharoni Z, Yanir Y, Keynan Y, Alfie Y, Halpern P. Underwater Hearing and Sound Localization with and without an Air Interface. Otology \& Neurotology : Official publication of the American Otological Society, American Neurotology Society [and] 
European Academy of Otology and Neurotology 2005; 26(1): 127-130. DOI: $10.1097 / 00129492-200501000-00023$
27 Jain K. Effects of Diving and High Pressure on the Human Body in Text book of Hyperbaric Medicine, 6th edition. Springer. Cham, Switzerland, 2017: 23-31. 